\title{
Ipilimumab-induced colitis: a rare but serious side
} effect
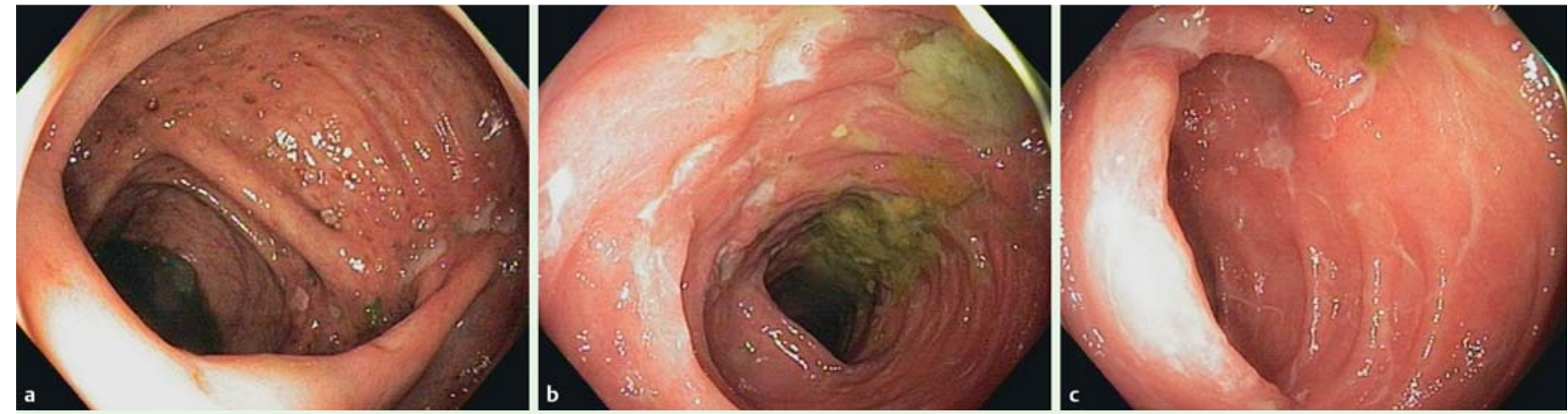

Fig. 1 Scattered ulcerations, patchy erythema, and diffuse edema throughout the colon as seen on colonoscopy: a cecum, b transverse colon, c sigmoid colon.

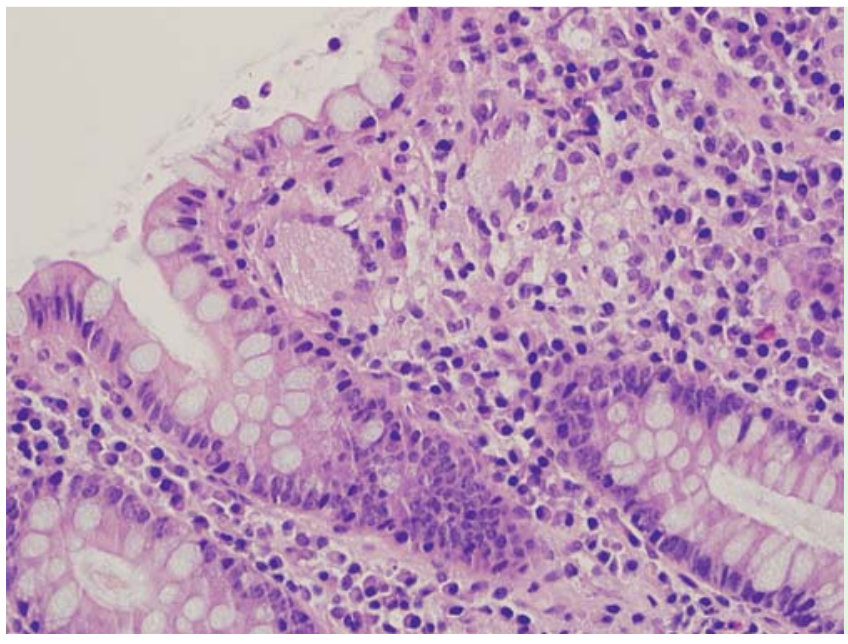

Fig. 2 Colonic mucosa with superficial granulomata comprised of epithelioid macrophages and a giant cell. The crypts show loss of the normal lamina propria gradient, with increased lymphoplasmacytic inflammation extending to the base.

Colitis is a serious condition with associated morbidity and mortality. Colitis may be caused by multiple agents, including ischemia, infection, irritable bowel disease, and medications. Ipilimumab, a monoclonal antibody used for the treatment of melanoma, has in rare cases been associated with colitis [1]. This case report describes one such rare case of ipilimumab-induced colitis.

A 73-year-old man with metastatic ocular melanoma presented to the hospital for diarrhea. For his melanoma, he was taking ipilimumab. A few weeks after his second cycle of ipilimumab, he began experiencing 7-10 watery stools daily. He was given loperamide by his primary physician without improvement in the diarrhea. Upon presentation at the hospital, his vital signs and physical exam were normal. His lab results revealed normocytic anemia and hypoalbuminemia $(2.6 \mathrm{~g} / \mathrm{dL})$. Stool studies were positive for the presence of fecal leukocytes but negative for Clostridium difficile toxin. Culture was also negative. The patient underwent CT of the abdomen, which showed known metastatic disease. He then underwent colonoscopy, which revealed scattered ulcerations with patchy erythema and diffuse edema extending from the cecum to the rectum ( $\bullet$ Fig. 1 ). Colon biopsies were obtained and showed mild active colitis with rare granulomata but no atypia or malignancy ( $\bullet$ Fig. 2). Acid-fast bacilli and fungal stains were negative. Given the history and findings, ipilimumab was discontinued and the patient started on intravenous methyl- prednisolone. Within 2 days, his diarrhea was significantly improved. He was discharged on oral prednisone. Two weeks after discharge, he was doing well with complete resolution of his diarrhea.

Multiple chemotherapy agents and monoclonal antibodies, such as ipilimumab, have been shown to cause diarrhea but rarely colitis [2]. Drug-induced colitis should always be part of the differential diagnosis for patients undergoing any form of chemotherapy who present with diarrhea or hematochezia. If colitis is secondary to ipilimumab, prompt discontinuation with initiation of corticosteroids may quickly reverse the active colitis, limit morbidity, and improve quality of life $[3,4]$.

Endoscopy_UCTN_Code_CCL_1AD_2AD

Competing interests: None

Alisha M. Hinds' ${ }^{1}$ Dina S. Ahmad ${ }^{1}$, Joseph E. Muenster ${ }^{1}$, Zachary M. Berg ${ }^{1}$, Kristi T. Lopez ${ }^{1}$, Jason S.L. Holly², Michelle L. Matteson-Kome', Matthew L. Bechtold'

${ }^{1}$ Department of Medicine, University of Missouri School of Medicine, Columbia, Missouri, United States of America

2 Department of Anatomical Pathology, University of Missouri School of Medicine, Columbia, Missouri, United States of America 


\section{References}

1 Acharya UH, Jeter JM. Use of ipilimumab in the treatment of melanoma. Clin Pharmacol 2013; 5 (Suppl. 01): 21-27

2 Stein A, Voigt W, Jordan K. Chemotherapyinduced diarrhea: pathophysiology, frequency and guideline-based management. Ther Adv Med Oncol 2010; 2: 51-63

3 Lyall A, Vargas HA, Carvajal RD et al. Ipilimumab-induced colitis on FDG PET/CT. Clin Nucl Med 2012; 37: 629-630

4 Minor DR, Chin K, Kashani-Sabet M. Infliximab in the treatment of anti-CTLA4 antibody (ipilimumab) induced immune-related colitis. Cancer Biother Radiopharm 2009; 24: $321-325$

\section{Bibliography}

DOI http://dx.doi.org/

10.1055/s-0034-1377209

Endoscopy 2014; 46: E308-E309

(c) Georg Thieme Verlag KG

Stuttgart · New York

ISSN 0013-726X

\section{Corresponding author}

Matthew L. Bechtold, MD

Division of Gastroenterology Five Hospital Drive, CE405

Columbia, MO 65212

United States of America

Fax: +1-573-884-4595

bechtoldm@health.missouri.edu 